IJMMS 30:1 (2002) 39-47

PII. S0161171202012097

http://ijmms.hindawi.com

(c) Hindawi Publishing Corp.

\title{
COHOMOLOGY OF THE VARIATIONAL COMPLEX \\ IN THE CLASS OF EXTERIOR FORMS OF FINITE JET ORDER
}

\section{GENNADI SARDANASHVILY}

Received 19 February 2001 and in revised form 18 September 2001

\begin{abstract}
We obtain the cohomology of the variational complex on the infinite-order jet space of a smooth fiber bundle in the class of exterior forms of finite jet order. In particular, this provides a solution of the global inverse problem of the calculus of variations of finite order on fiber bundles.
\end{abstract}

2000 Mathematics Subject Classification: 55N30, 58A20, 58E30.

1. Introduction. Let $Y \rightarrow X$ be a smooth fiber bundle; put further $\operatorname{dim} X=n \geq 1$. We obtain cohomology of the variational complex on the infinite-order jet space $J^{\infty} Y$ of $Y \rightarrow X$ in the class of exterior forms of finite jet order. This is cohomology of the horizontal (or total) differential $d_{H}$ and the variational operator $\delta$.

We usually consider two bigraded differential algebras $\mathscr{T}_{\infty}^{*}$ and $\mathfrak{O}_{\infty}^{*}$ of exterior forms on $J^{\infty} Y$. The algebra $\mathscr{T}_{\infty}^{*}$ consists of exterior forms which are locally the pullback of exterior forms on finite-order jet manifolds. The de Rham cohomology, $d_{H^{-}}$and $\delta$ cohomology of $\mathcal{T}_{\infty}^{*}$ have been investigated in [2, 12]. The subalgebra $\mathcal{O}_{\infty}^{*}$ of $\mathscr{T}_{\infty}^{*}$ consists of the pullback of exterior forms on finite-order jet manifolds. Cohomology of $0_{\infty}^{*}$, except the de Rham cohomology and the particular result of [14] on $\delta$-cohomology, remains unknown. At the same time, the algebra $0_{\infty}^{*}$ is most interesting for applications. We prove that the graded differential algebra $0_{\infty}^{*}$ has the same $d_{H^{-}}$and $\delta$-cohomology as $\mathscr{T}_{\infty}^{*}$ (see Theorem 5.1 below). In particular, this result provides a solution of the global inverse problem of the calculus of variations of finite order on fiber bundles (see Corollary 6.1 below), and is also applied to computation of BRST cohomology in quantum field theory [6].

Note that a solution of the global inverse problem of the calculus of variations in the class of exterior forms of bounded jet order has been suggested in [2] by a computation of cohomology of a fixed order variational sequence. However, this computation requires rather sophisticated ad hoc technique in order to be reproduced (see $[9,10,15]$ for a different variational sequence). Our results agree with those in [2], but without minimizing an order of a Lagrangian.

The article is organized as follows. Section 2 is devoted to differential calculus on $J^{\infty} Y$. In Section 3, the variational bicomplex on $J^{\infty} Y$ is set. In Section 4, cohomology of the graded differential algebra $\mathscr{T}_{\infty}^{*}$ is computed. In Section 5, an isomorphism of cohomology of $\mathscr{T}_{\infty}^{*}$ to that of $\mathcal{O}_{\infty}^{*}$ is proved. In Section 6 , a solution of the global inverse 
problem of the calculus of variations in the class of exterior forms of finite jet order is provided.

2. The differential calculus on $J^{\infty} Y$. Smooth manifolds throughout are assumed to be real, finite-dimensional, Hausdorff, second-countable (i.e., paracompact), and connected. We follow the terminology of $[4,8]$, where a sheaf $S$ is a particular topological bundle, $\bar{S}$ denotes the canonical presheaf of sections of the sheaf $S$, and $\Gamma(S)$ is the group of global sections of $S$.

Recall that the infinite-order jet space $J^{\infty} Y$ of a smooth fiber bundle $Y \rightarrow X$ is defined as a projective limit of the inverse system

$$
X \stackrel{\pi}{\longleftarrow} Y \stackrel{\pi_{0}^{1}}{\longleftarrow} \cdots \longleftarrow J^{r-1} Y \stackrel{\pi_{r-1}^{r}}{\longleftarrow} J^{r} Y \longleftarrow \cdots
$$

of finite-order jet manifolds $J^{r} Y$ of $Y \rightarrow X$. Endowed with the projective limit topology, $J^{\infty} Y$ is a paracompact Fréchet manifold [12]. A bundle coordinate atlas $\left\{U_{Y},\left(x^{\lambda}, y^{i}\right)\right\}$ of $Y \rightarrow X$ yields the manifold coordinate atlas

$$
\left\{\left(\pi_{0}^{\infty}\right)^{-1}\left(U_{Y}\right),\left(x^{\lambda}, y_{\Lambda}^{i}\right)\right\}, \quad 0 \leq|\Lambda|,
$$

of $J^{\infty} Y$, together with the transition functions

$$
y_{\lambda+\Lambda}^{\prime i}=\frac{\partial x^{\mu}}{\partial x^{\prime \lambda}} d_{\mu} y_{\Lambda}^{\prime i},
$$

where $\Lambda=\left(\lambda_{k} \cdots \lambda_{1}\right), \lambda+\Lambda=\left(\lambda \lambda_{k} \cdots \lambda_{1}\right)$ are multi-indices and $d_{\lambda}$ denotes the total derivative

$$
d_{\lambda}=\partial_{\lambda}+\sum_{|\Lambda| \geq 0} y_{\lambda+\Lambda}^{i} \partial_{i}^{\Lambda}
$$

With the inverse system (2.1), we have the direct system

$$
\mathbb{O}^{*}(X) \stackrel{\pi^{*}}{\longrightarrow} \mathbb{O}_{0}^{*} \stackrel{\pi_{0}^{1 *}}{\longrightarrow} \mathbb{O}_{1}^{*} \stackrel{\pi_{1}^{2 *}}{\longrightarrow} \cdots \stackrel{\pi_{r-1}^{r} *}{\longrightarrow} \mathcal{O}_{r}^{*} \longrightarrow \cdots
$$

of graded differential $\mathbb{R}$-algebras $\mathrm{O}_{r}^{*}$ of exterior forms on finite-order jet manifolds $J^{r} Y$, where $\pi_{r-1}^{r}$ * are the pullback monomorphisms. The direct limit of this direct system is the above mentioned graded differential algebra $\mathrm{O}_{\infty}^{*}$ of exterior forms on finite-order jet manifolds modulo the pullback identification. By passing to the direct limit of the de Rham complexes of exterior forms on finite-order jet manifolds, the de Rham cohomology of $O_{\infty}^{*}$ has been proved to coincide with the de Rham cohomology of the fiber bundle $Y[1,3]$. However, this is not a way of studying other cohomologies of the algebra $\mathcal{O}_{\infty}^{*}$. To solve this problem, we enlarge $\mathcal{O}_{\infty}^{*}$ as follows.

Let $\Theta_{r}^{*}$ be a sheaf of germs of exterior forms on the $r$-order jet manifold $J^{r} Y$, and $\overline{\mathcal{C}}_{r}^{*}$ its canonical presheaf. There is the direct system of canonical presheaves

$$
\overline{\mathfrak{\oplus}}_{X}^{*} \stackrel{\pi^{*}}{\longrightarrow} \bar{\oplus}_{0}^{*} \stackrel{\pi_{0}^{1 *}}{\longrightarrow} \overline{\mathscr{\oplus}}_{1}^{*} \stackrel{\pi_{1}^{2 *}}{\longrightarrow} \cdots \stackrel{\pi_{r-1}^{r} *}{\longrightarrow} \overline{\mathscr{\oplus}}_{r}^{*} \longrightarrow \cdots,
$$

where $\pi_{r-1}^{r} *$ are the pullback monomorphisms. Its direct limit $\overline{\mathscr{G}}_{\infty}^{*}$ is a presheaf of graded differential $\mathbb{R}$-algebras on $J^{\infty} Y$. Let $\mathbb{C}_{\infty}^{*}$ be a sheaf constructed from $\overline{\mathfrak{G}}_{\infty}^{*}, \overline{\mathbb{C}}_{\infty}^{*}$ 
its canonical presheaf, and $\mathscr{T}_{\infty}^{*}=\Gamma\left(\overleftarrow{\nearrow}_{\infty}^{*}\right)$ the structure algebra of sections of the sheaf $\mathbb{C}_{\infty}^{*}$. There are $\mathbb{R}$-algebra monomorphisms $\overline{\mathcal{G}}_{\infty}^{*} \rightarrow \overline{\mathbb{C}}_{\infty}^{*}$ and $\mathcal{O}_{\infty}^{*} \rightarrow \mathcal{T}_{\infty}^{*}$. The key point is that, since the paracompact space $J^{\infty} Y$ admits a partition of unity by elements of the ring $\mathscr{T}_{\infty}^{0}$ [12], the sheaves of $\mathscr{T}_{\infty}^{0}$-modules on $J^{\infty} Y$ are fine and, consequently, acyclic. Therefore, the abstract de Rham theorem on cohomology of a sheaf resolution [8] can be called into play in order to obtain cohomology of the graded differential algebra $\mathscr{T}_{\infty}^{*}$. Then we prove that $\mathcal{O}_{\infty}^{*}$ has the same $d_{H^{-}}$and $\delta$-cohomology as $\mathscr{T}_{\infty}^{*}$.

For short, we agree to call elements of $\mathscr{T}_{\infty}^{*}$ the exterior forms on $J^{\infty} Y$. Restricted to a coordinate chart $\left(\pi_{0}^{\infty}\right)^{-1}\left(U_{Y}\right)$ of $J^{\infty} Y$, they can be written in a coordinate form, where horizontal forms $\left\{d x^{\lambda}\right\}$ and contact 1-forms $\left\{\theta_{\Lambda}^{i}=d y_{\Lambda}^{i}-y_{\lambda+\Lambda}^{i} d x^{\lambda}\right\}$ provide local generators of the algebra $\mathscr{T}_{\infty}^{*}$. There is the canonical decomposition

$$
\mathscr{T}_{\infty}^{*}=\bigoplus_{k, s} \mathscr{T}_{\infty}^{k, s}, \quad 0 \leq k, 0 \leq s \leq n,
$$

of $\mathscr{T}_{\infty}^{*}$ into $\mathscr{T}_{\infty}^{0}$-modules $\mathscr{T}_{\infty}^{k, s}$ of $k$-contact and $s$-horizontal forms, together with the corresponding projections

$$
h_{k}: \mathscr{T}_{\infty}^{*} \longrightarrow \mathscr{T}_{\infty}^{k, *}, \quad 0 \leq k, \quad h^{s}: \mathscr{T}_{\infty}^{*} \longrightarrow \mathscr{T}_{\infty}^{*, s}, \quad 0 \leq s \leq n .
$$

Accordingly, the exterior differential on $\mathscr{T}_{\infty}^{*}$ is split into the sum $d=d_{H}+d_{V}$ of horizontal and vertical differentials such that,

$$
\begin{array}{ll}
d_{H} \circ h_{k}=h_{k} \circ d \circ h_{k}, & d_{H}(\phi)=d x^{\lambda} \wedge d_{\lambda}(\phi), \\
d_{V} \circ h^{s}=h^{s} \circ d \circ h^{s}, & d_{V}(\phi)=\theta_{\Lambda}^{i} \wedge \partial_{i}^{\Lambda} \phi, \quad \phi \in \mathcal{T}_{\infty}^{*} .
\end{array}
$$

3. The variational bicomplex. Being nilpotent, the differentials $d_{V}$ and $d_{H}$ provide the natural bicomplex $\left\{\mathfrak{t}_{\infty}^{k, m}\right\}$ of the sheaf $\mathfrak{t}_{\infty}^{*}$ on $J^{\infty} Y$. To complete it to the variational bicomplex, we define the projection $\mathbb{R}$-module endomorphism:

$$
\begin{aligned}
\tau & =\sum_{k>0} \frac{1}{k} \bar{\tau} \circ h_{k} \circ h^{n}, \\
\bar{\tau}(\phi) & \left.=(-1)^{|\Lambda|} \theta^{i} \wedge\left[d_{\Lambda}\left(\partial_{i}^{\Lambda}\right\rfloor \phi\right)\right], \quad 0 \leq|\Lambda|, \phi \in \bar{\oplus}_{\infty}^{>0, n},
\end{aligned}
$$

of $\bar{\oplus}_{\infty}^{*}$ such that

$$
\tau \circ d_{H}=0, \quad \tau \circ d \circ \tau-\tau \circ d=0 .
$$

Introduced on elements of the presheaf $\bar{\oplus}_{\infty}^{*}(\mathrm{cf} .[3,5,13])$, this endomorphism is induced on the sheaf $\mathbb{C}_{\infty}^{*}$ and its structure algebra $\mathscr{T}_{\infty}^{*}$. Put

$$
\mathfrak{E}_{k}=\tau\left(\mathbb{T}_{\infty}^{k, n}\right), \quad E_{k}=\tau\left(\mathscr{T}_{\infty}^{k, n}\right), \quad k>0 .
$$

Since $\tau$ is a projection operator, we have isomorphisms

$$
\overline{\mathfrak{E}}_{k}=\tau\left(\overline{\mathbb{T}}_{\infty}^{k, n}\right), \quad E_{k}=\Gamma\left(\mathfrak{E}_{k}\right) .
$$

The variational operator on $\mathbb{t}_{\infty}^{*, n}$ is defined as the morphism $\delta=\tau \circ d$. It is nilpotent, and obeys the relation

$$
\delta \circ \tau-\tau \circ d=0
$$


Let $\mathbb{R}$ and $\odot_{X}^{*}$ denote the constant sheaf on $J^{\infty} Y$ and the sheaf of exterior forms on $X$, respectively. The operators $d_{V}, d_{H}, \tau$, and $\delta$ give the following variational bicomplex of sheaves of differential forms on $J^{\infty} Y$ :

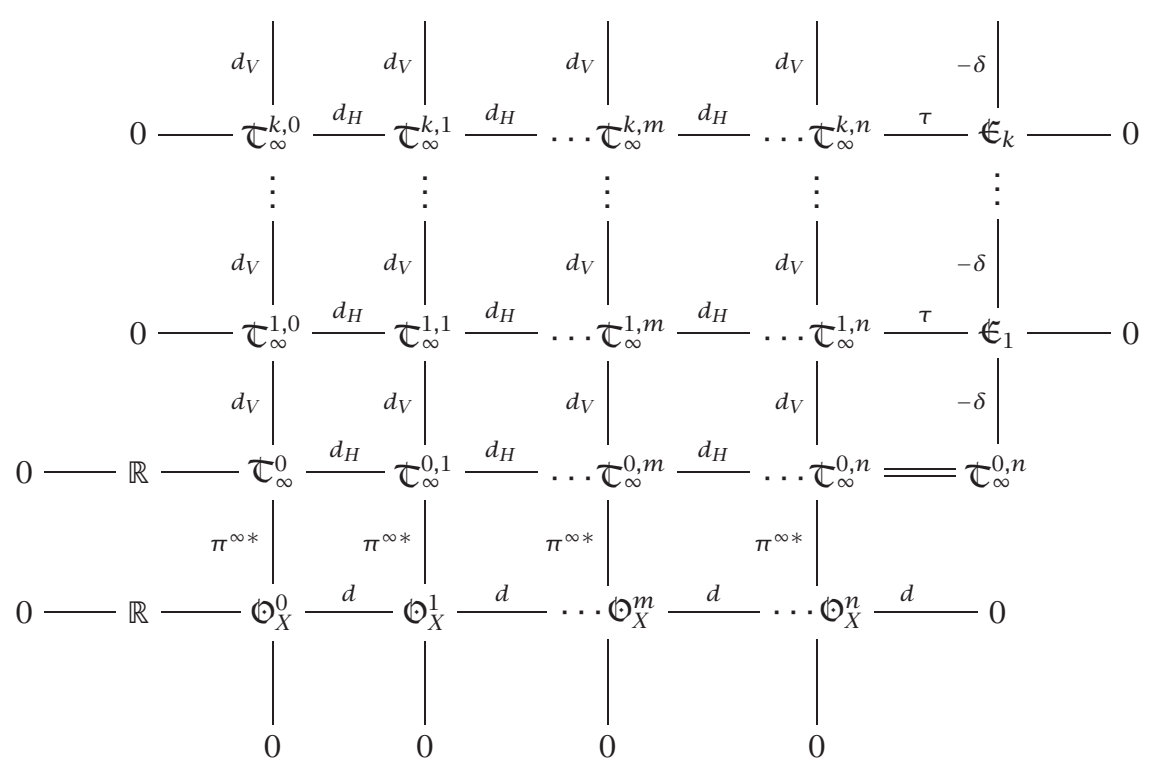

The second row and the last column of this bicomplex form the variational complex

$$
0 \longrightarrow \mathbb{R} \longrightarrow \mathbb{T}_{\infty}^{0} \stackrel{d_{H}}{\longrightarrow} \mathbb{t}_{\infty}^{0,1} \stackrel{d_{H}}{\longrightarrow} \cdots \stackrel{d_{H}}{\longrightarrow} \mathbb{T}_{\infty}^{0, n} \stackrel{\delta}{\longrightarrow} \mathfrak{C}_{1} \stackrel{\delta}{\longrightarrow} \mathfrak{E}_{2} \longrightarrow \cdots
$$

The corresponding variational bicomplexes and variational complexes of graded differential algebras $\mathscr{T}_{\infty}^{*}$ and $\mathcal{O}_{\infty}^{*}$ take place.

There are the well-known statements summarized usually as the algebraic Poincaré lemma (cf. [11, 13]).

LEMMA 3.1. If $Y$ is a contactible bundle $\mathbb{R}^{n+p} \rightarrow \mathbb{R}^{n}$, the variational bicomplex of the graded differential algebra $\mathrm{O}_{\infty}^{*}$ is exact.

It follows that the variational bicomplex (3.6) and, consequently, the variational complex (3.7) are exact for any smooth bundle $Y \rightarrow X$. Moreover, the sheaves $\mathbb{C}_{\infty}^{k, m}$ in this bicomplex are fine, and so are the sheaves $\mathfrak{E}_{k}$ in accordance with the following lemma.

LEMMA 3.2. Sheaves $\mathfrak{\in}_{k}$ are fine.

Proof. Although the $\mathbb{R}$-modules $E_{k>1}$ fail to be $\mathscr{T}_{\infty}^{0}$-modules [13], we can use the fact that the sheaves $\mathfrak{E}_{k>0}$ are projections $\tau\left(\overleftarrow{C}_{\infty}^{k, n}\right)$ of sheaves of $\mathscr{T}_{\infty}^{0}$-modules. Let $\left\{U_{i}\right\}_{i \in I}$ be a locally finite open covering of $J^{\infty} Y$, and let $\left\{f_{i} \in \mathscr{T}_{\infty}^{0}\right\}$ be the associated partition of unity. For any open subset $U \subset J^{\infty} Y$ and any section $\varphi$ of the sheaf $\mathbb{C}_{\infty}^{k, n}$ 
over $U$, put $h_{i}(\varphi)=f_{i} \varphi$. The endomorphisms $h_{i}$ of $\mathfrak{t}_{\infty}^{k, n}$ yield the $\mathbb{R}$-module endomorphisms

$$
\bar{h}_{i}=\tau \circ h_{i}: \mathfrak{E}_{k} \stackrel{\text { in }}{\longrightarrow} \mathbb{T}_{\infty}^{k, n} \stackrel{h_{i}}{\longrightarrow} \mathbb{T}_{\infty}^{k, n} \stackrel{\tau}{\longrightarrow} \mathfrak{E}_{k}
$$

of the sheaves $\mathfrak{E}_{k}$. They possess the properties required for $\mathfrak{E}_{k}$ to be a fine sheaf. Indeed, for each $i \in I, \operatorname{supp} f_{i} \subset U_{i}$ provides a closed set such that $\bar{h}_{i}$ is zero outside this set, while the $\operatorname{sum} \sum_{i \in I} \bar{h}_{i}$ is the identity morphism.

Thus, the columns and rows of bicomplex (3.6) as well as the variational complex (3.7) are sheaf resolutions, and the abstract de Rham theorem can be applied to them. Here, we restrict our consideration to the variational complex.

4. Cohomology of $\mathcal{T}_{\infty}^{*}$. The variational complex (3.7) is a resolution of the constant sheaf $\mathbb{R}$ on $J^{\infty} Y$. We start from the following lemma.

LEMMA 4.1. There is an isomorphism

$$
H^{*}\left(J^{\infty} Y, \mathbb{R}\right)=H^{*}(Y, \mathbb{R})=H^{*}(Y)
$$

between cohomology $H^{*}\left(J^{\infty} Y, \mathbb{R}\right)$ of $J^{\infty} Y$ with coefficients in the constant sheaf $\mathbb{R}$, that $H^{*}(Y, \mathbb{R})$ of $Y$, and the de Rham cohomology $H^{*}(Y)$ of $Y$.

Proof. Since $Y$ is a strong deformation retract of $J^{\infty} Y$ [1], the first isomorphism in (4.1) follows from the Vietoris-Begle theorem [4], while the second one results from the familiar de Rham theorem.

Consider the de Rham complex of sheaves

$$
0 \rightarrow \mathbb{R} \longrightarrow \mathbb{t}_{\infty}^{0} \stackrel{d}{\longrightarrow} \mathbb{t}_{\infty}^{1} \stackrel{d}{\longrightarrow} \cdots
$$

on $J^{\infty} Y$ and the corresponding de Rham complex of their structure algebras

$$
0 \longrightarrow \mathbb{R} \longrightarrow \mathscr{T}_{\infty}^{0} \stackrel{d}{\longrightarrow} \mathscr{T}_{\infty}^{1} \stackrel{d}{\longrightarrow} \cdots
$$

Complex (4.2) is exact due to the Poincaré lemma, and is a resolution of the constant sheaf $\mathbb{R}$ on $J^{\infty} Y$ since sheaves $\mathbb{t}_{\infty}^{r}$ are fine. Then, the abstract de Rham theorem and Lemma 4.1 lead to the following.

Proposition 4.2. The de Rham cohomology $H^{*}\left(\mathcal{T}_{\infty}^{*}\right)$ of the graded differential algebra $\mathscr{T}_{\infty}^{*}$ is isomorphic to that $H^{*}(Y)$ of the bundle $Y$.

It follows that every closed form $\phi \in \mathscr{T}_{\infty}^{*}$ is split into the sum

$$
\phi=\varphi+d \xi, \quad \xi \in \mathscr{T}_{\infty}^{*},
$$

where $\varphi$ is a closed form on the fiber bundle $Y$.

Similarly, from the abstract de Rham theorem and Lemma 4.1, we obtain the following. 
Proposition 4.3. There is an isomorphism between $d_{H}$ - and $\delta$-cohomology of the variational complex

$$
0 \longrightarrow \mathbb{R} \longrightarrow \mathscr{T}_{\infty}^{0} \stackrel{d_{H}}{\longrightarrow} \mathscr{T}_{\infty}^{0,1} \stackrel{d_{H}}{\longrightarrow} \cdots \stackrel{d_{H}}{\longrightarrow} \mathscr{T}_{\infty}^{0, n} \stackrel{\delta}{\longrightarrow} E_{1} \stackrel{\delta}{\longrightarrow} E_{2} \longrightarrow \cdots
$$

and the de Rham cohomology of the fiber bundle $Y$, namely,

$$
H^{k<n}\left(d_{H} ; \mathscr{T}_{\infty}^{*}\right)=H^{k<n}(Y), \quad H^{k-n}\left(\delta ; \mathcal{T}_{\infty}^{*}\right)=H^{k \geq n}(Y)
$$

This isomorphism recovers the results of $[2,12]$, but notes also the following. Relation (3.5) for $\tau$ and relation $h_{0} d=d_{H} h_{0}$ for $h_{0}$ define a homomorphism of the de Rham complex (4.3) of the algebra $\mathscr{T}_{\infty}^{*}$ to its variational complex (4.5). The corresponding homomorphism of their cohomology groups is an isomorphism by virtue of Propositions 4.2 and 4.3. Then, splitting (4.4) leads to the following decompositions.

Proposition 4.4. Any $d_{H}$-closed form $\sigma \in \mathscr{T}^{0, m}, m<n$, is represented by a sum

$$
\sigma=h_{0} \varphi+d_{H} \xi, \quad \xi \in \mathscr{T}_{\infty}^{m-1}
$$

where $\varphi$ is a closed $m$-form on $Y$. Any $\delta$-closed form $\sigma \in \mathscr{T}^{k, n}, k \geq 0$, is split into

$$
\begin{array}{ll}
\sigma=h_{0} \varphi+d_{H} \xi, & k=0, \xi \in \mathscr{T}_{\infty}^{0, n-1}, \\
\sigma=\tau(\varphi)+\delta(\xi), & k=1, \xi \in \mathscr{T}_{\infty}^{0, n}, \\
\sigma=\tau(\varphi)+\delta(\xi), & k>1, \xi \in E_{k-1},
\end{array}
$$

where $\varphi$ is a closed $(n+k)$-form on $Y$.

\section{Cohomology of $\mathcal{O}_{\infty}^{*}$}

THEOREM 5.1. Graded differential algebra $\mathcal{O}_{\infty}^{*}$ has the same $d_{H^{-}}$and $\delta$-cohomology as $\mathscr{T}_{\infty}^{*}$.

Proof. Let the common symbol $D$ stand for $d_{H}$ and $\delta$. Bearing in mind the decompositions (4.7), (4.8), (4.9), and (4.10), it suffices to show that, if an element $\phi \in \mathcal{O}_{\infty}^{*}$ is $D$-exact in the algebra $\mathcal{T}_{\infty}^{*}$, then it is so in the algebra $\mathcal{O}_{\infty}^{*}$. Lemma 3.1 states that, if $Y$ is a contractible bundle and a $D$-exact form $\phi$ on $J^{\infty} Y$ is of finite jet order $[\phi]$ (i.e., $\left.\phi \in \mathbb{O}_{\infty}^{*}\right)$, there exists an exterior form $\varphi \in \mathbb{O}_{\infty}^{*}$ on $J^{\infty} Y$ such that $\phi=D \varphi$. Moreover, a glance at the homotopy operators for $d_{H}$ and $\delta$ (see [11]) shows that the jet order $[\varphi]$ of $\varphi$ is bounded by an integer $N([\phi])$, depending only on the jet order of $\phi$. We call this fact the finite exactness of the operator $D$. Given an arbitrary bundle $Y$, the finite exactness takes place on $\left.J^{\infty} Y\right|_{U}$ over any domain (i.e., a contractible open subset) $U \subset Y$. We prove the following:

(i) given a family $\left\{U_{\alpha}\right\}$ of disjoint open subsets of $Y$, suppose that the finite exactness takes place on $\left.J^{\infty} Y\right|_{U_{\alpha}}$ over every subset $U_{\alpha}$ from this family. Then, it is true on $J^{\infty} Y$ over the union $\bigcup_{\alpha} U_{\alpha}$ of these subsets;

(ii) suppose that the finite exactness of the operator $D$ takes place on $J^{\infty} Y$ over open subsets $U, V$ of $Y$ and their nonempty overlap $U \cap V$. Then, it is also true on $\left.J^{\infty} Y\right|_{U \cup V}$. 
Proof of (i). Let $\phi \in \mathcal{O}_{\infty}^{*}$ be a $D$-exact form on $J^{\infty} Y$. The finite exactness on $\left(\pi_{0}^{\infty}\right)^{-1}\left(\cup U_{\alpha}\right)$ holds since $\phi=D \varphi_{\alpha}$ on every $\left(\pi_{0}^{\infty}\right)^{-1}\left(U_{\alpha}\right)$ and $\left[\varphi_{\alpha}\right]<N([\phi])$.

Proof OF (ii). Let $\phi=D \varphi \in 0_{\infty}^{*}$ be a $D$-exact form on $J^{\infty} Y$. By assumption, it can be brought into the form $D \varphi_{U}$ on $\left(\pi_{0}^{\infty}\right)^{-1}(U)$ and $D \varphi_{V}$ on $\left(\pi_{0}^{\infty}\right)^{-1}(V)$, where $\varphi_{U}$ and $\varphi_{V}$ are exterior forms of bounded jet order. We consider their difference $\varphi_{U}-\varphi_{V}$ on $\left(\pi_{0}^{\infty}\right)^{-1}(U \cap V)$. It is a $D$-exact form of bounded jet order $\left[\varphi_{U}-\varphi_{V}\right]<N([\phi])$ which, by assumption, can be written as $\varphi_{U}-\varphi_{V}=D \sigma$ where $\sigma$ is also of bounded jet order $[\sigma]<N(N([\phi]))$. Lemma 5.2 below shows that $\sigma=\sigma_{U}+\sigma_{V}$ where $\sigma_{U}$ and $\sigma_{V}$ are exterior forms of bounded jet order on $\left(\pi_{0}^{\infty}\right)^{-1}(U)$ and $\left(\pi_{0}^{\infty}\right)^{-1}(V)$, respectively. Then, putting

$$
\left.\varphi^{\prime}\right|_{U}=\varphi_{U}-D \sigma_{U},\left.\quad \varphi^{\prime}\right|_{V}=\varphi_{V}+D \sigma_{V}
$$

we have the form $\phi$, equal to $D \varphi_{U}^{\prime}$ on $\left(\pi_{0}^{\infty}\right)^{-1}(U)$ and $D \varphi_{V}^{\prime}$ on $\left(\pi_{0}^{\infty}\right)^{-1}(V)$, respectively. Since the difference $\varphi_{U}^{\prime}-\varphi_{V}^{\prime}$ on $\left(\pi_{0}^{\infty}\right)^{-1}(U \cap V)$ vanishes, we obtain $\phi=D \varphi^{\prime}$ on $\left(\pi_{0}^{\infty}\right)^{-1}(U \cup V)$ where

$$
\varphi^{\prime} \stackrel{\text { def }}{=}\left\{\begin{array}{l}
\left.\varphi^{\prime}\right|_{U}=\varphi_{U}^{\prime}, \\
\left.\varphi^{\prime}\right|_{V}=\varphi_{V}^{\prime}
\end{array}\right.
$$

is of bounded jet order $\left[\varphi^{\prime}\right]<N(N([\phi]))$. To prove the finite exactness of $D$ on $J^{\infty} Y$, it remains to choose an appropriate cover of $Y$. A smooth manifold $Y$ admits a countable cover $\left\{U_{\xi}\right\}$ by domains $U_{\xi}, \xi \in \mathbb{N}$, and its refinement $\left\{U_{i j}\right\}$, where $j \in \mathbb{N}$ and $i$ runs through a finite set, such that $U_{i j} \cap U_{i k}=\varnothing, j \neq k$ [7]. Then $Y$ has a finite cover $\left\{U_{i}=\bigcup_{j} U_{i j}\right\}$. Since the finite exactness of the operator $D$ takes place over any domain $U_{\xi}$, it also holds over any member $U_{i j}$ of the refinement $\left\{U_{i j}\right\}$ of $\left\{U_{\xi}\right\}$ and, in accordance with Theorem 5.1 item (i) above, over any member of the finite cover $\left\{U_{i}\right\}$ of $Y$. Then by virtue of item (ii) above, the finite exactness of $D$ takes place over $Y$.

LEMMA 5.2. Let $U$ and $V$ be open subsets of a bundle $Y$ and $\sigma \in \oplus_{\infty}^{*}$, an exterior form of bounded jet order on $\left(\pi_{0}^{\infty}\right)^{-1}(U \cap V) \subset J^{\infty} Y$. Then, $\sigma$ is split into a sum $\sigma_{U}+$ $\sigma_{V}$ of exterior forms $\sigma_{U}$ and $\sigma_{V}$ of bounded jet order on $\left(\pi_{0}^{\infty}\right)^{-1}(U)$ and $\left(\pi_{0}^{\infty}\right)^{-1}(V)$, respectively.

Proof. By taking a smooth partition of unity on $U \cup V$ subordinate to the cover $\{U, V\}$ and passing to the function with support in $V$, we get a smooth real function $f$ on $U \cup V$ which is 0 on a neighborhood of $U-V$, and 1 on a neighborhood of $V-U$ in $U \cup V$. Let $\left(\pi_{0}^{\infty}\right)^{*} f$ be the pullback of $f$ onto $\left(\pi_{0}^{\infty}\right)^{-1}(U \cup V)$. The exterior form $\left(\left(\pi_{0}^{\infty}\right)^{*} f\right) \sigma$ is 0 on a neighborhood of $\left(\pi_{0}^{\infty}\right)^{-1}(U)$ and, therefore, can be extended by 0 to $\left(\pi_{0}^{\infty}\right)^{-1}(U)$. We denote it by $\sigma_{U}$. Accordingly, the exterior form $\left(1-\left(\pi_{0}^{\infty}\right)^{*} f\right) \sigma$ has an extension $\sigma_{V}$ by 0 to $\left(\pi_{0}^{\infty}\right)^{-1}(V)$. Then, $\sigma=\sigma_{U}+\sigma_{V}$ is a desired decomposition because $\sigma_{U}$ and $\sigma_{V}$ are of the jet order which does not exceed that of $\sigma$.

6. The global inverse problem. Expressions (4.8) and (4.9) in Proposition 4.4 provide a solution of the global inverse problem of the calculus of variations on fiber bundles in the class of Lagrangians $L \in \mathscr{T}_{\infty}^{0, n}$ of locally finite order [2, 12] (which is not 
so interesting for physical applications). These expressions together with Theorem 5.1 give a solution of the global inverse problem of the finite-order calculus of variations.

COROLLARY 6.1. (i) A finite-order Lagrangian $L \in 0_{\infty}^{0, n}$ is variationally trivial, that is, $\delta(L)=0$ if and only if

$$
L=h_{0} \varphi+d_{H} \xi, \quad \xi \in 0_{\infty}^{0, n-1}
$$

where $\varphi$ is a closed $n$-form on $Y$.

(ii) A finite-order Euler-Lagrange-type operator satisfies the Helmholtz condition $\delta(\mathscr{E})=0$ if and only if

$$
\mathscr{E}=\delta(L)+\tau(\phi), \quad L \in \mathbb{O}_{\infty}^{0, n},
$$

where $\phi$ is a closed $(n+1)$-form on $Y$.

Note that item (i) in Corollary 6.1 contains the result of [14]. As was mentioned above, the theses of Corollary 6.1 also agree with those of [2], but the proof of Theorem 5.1 does not give a sharp bound on the order of a Lagrangian.

\section{REFERENCES}

[1] I. M. Anderson, Introduction to the variational bicomplex, Mathematical Aspects of Classical Field Theory (Seattle, Wash., 1991), American Mathematical Society, Rhode Island, 1992, pp. 51-73.

[2] I. M. Anderson and T. Duchamp, On the existence of global variational principles, Amer. J. Math. 102 (1980), 781-868.

[3] M. Bauderon, Differential geometry and Lagrangian formalism in the calculus of variations, Differential Geometry, Calculus of Variations, and Their Applications, Lecture Notes in Pure and Appl. Math., vol. 100, Dekker, New York, 1985, pp. 67-82.

[4] G. E. Bredon, Sheaf Theory, McGraw-Hill, New York, 1967.

[5] G. Giachetta, L. Mangiarotti, and G. Sardanashvily, New Lagrangian and Hamiltonian Methods in Field Theory, World Scientific, Singapore, 1997.

[6] _ Iterated BRST cohomology, Lett. Math. Phys. 53 (2000), 143-156.

[7] W. Greub, S. Halperin, and R. Vanstone, Connections, Curvature, and Cohomology. Vol. I: de Rham Cohomology of Manifolds and Vector Bundles, Academic Press, New York, 1972.

[8] F. Hirzebruch, Topological Methods in Algebraic Geometry, Springer-Verlag, New York, 1966.

[9] D. Krupka, Variational sequences on finite order jet spaces, Differential Geometry and Its Applications (Brno, 1989), World Scientific Publishing, New Jersey, 1990, pp. 236254.

[10] D. Krupka and J. Musilová, Trivial Lagrangians in field theory, Differential Geom. Appl. 9 (1998), 293-305.

[11] P. J. Olver, Applications of Lie Groups to Differential Equations, Springer-Verlag, New York, 1993.

[12] F. Takens, A global version of the inverse problem of the calculus of variations, J. Differential Geom. 14 (1979), 543-562.

[13] W. M. Tulczyjew, The Euler-Lagrange resolution, Differential Geometrical Methods in Mathematical Physics (Proc. Conf., Aix-en-Provence/Salamanca, 1979), Lecture Notes in Math., vol. 836, Springer, Berlin, 1980, pp. 22-48.

[14] A. M. Vinogradov, The $\mathscr{b}$-spectral sequence, Lagrangian formalism, and conservation laws. II. The nonlinear theory, J. Math. Anal. Appl. 100 (1984), 41-129. 
[15] R. Vitolo, Finite order variational bicomplexes, Math. Proc. Cambridge Philos. Soc. 125 (1999), 321-333.

Gennadi Sardanashvily: Department of Theoretical Physics, Moscow State UniverSITY, 117234 MOSCOW, RUSSIA

E-mail address: sard@grav.phys.msu.su 


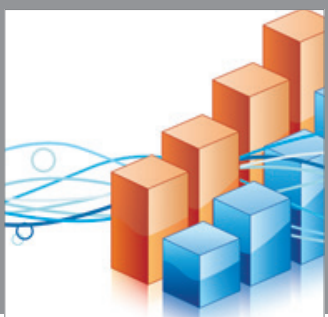

Advances in

Operations Research

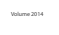

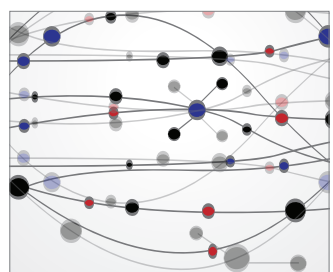

\section{The Scientific} World Journal
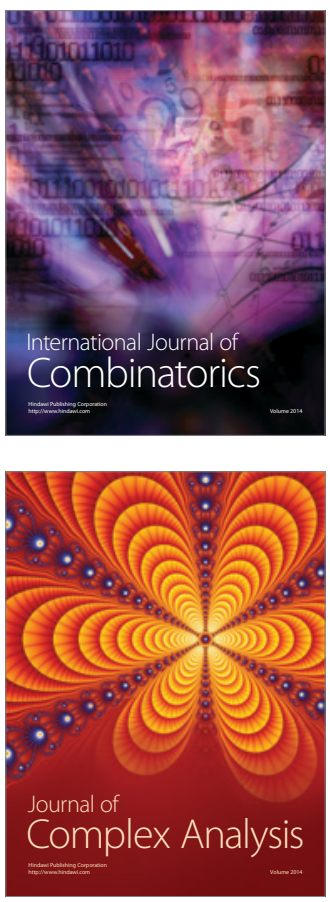

International Journal of

Mathematics and

Mathematical

Sciences
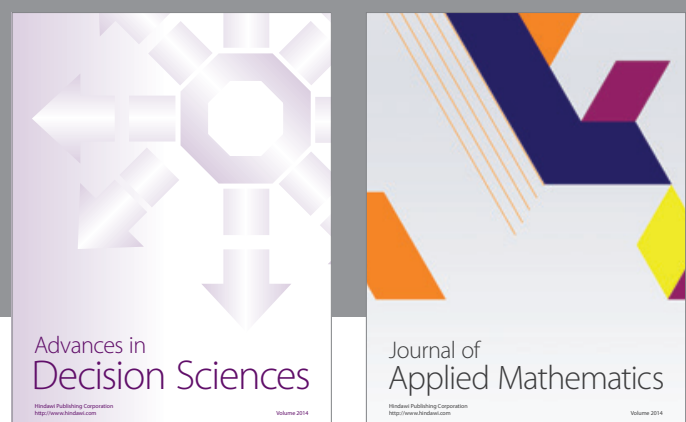

Journal of

Applied Mathematics
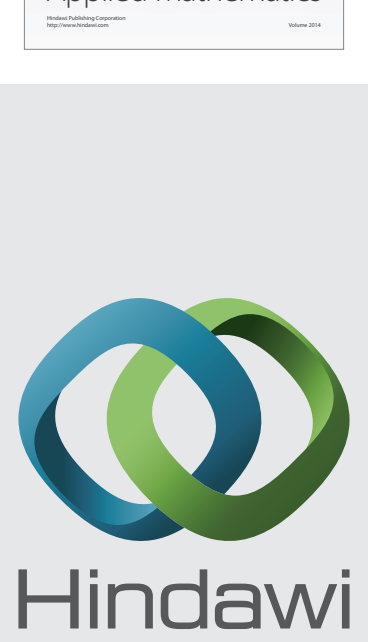

Submit your manuscripts at http://www.hindawi.com


Mathematical Problems in Engineering
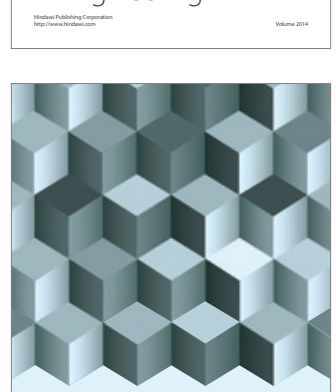

Journal of

Function Spaces
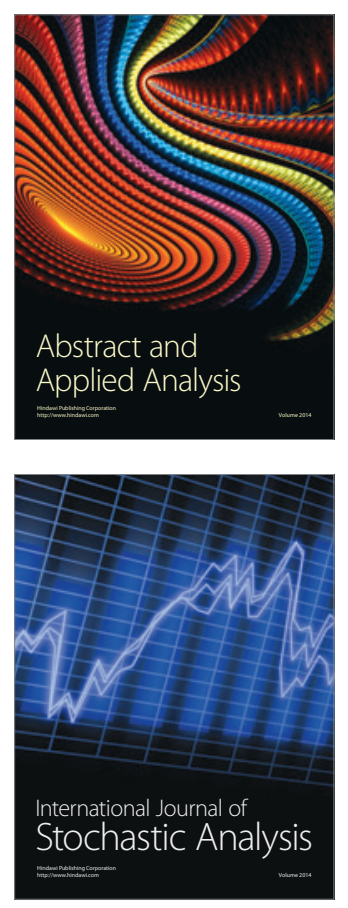

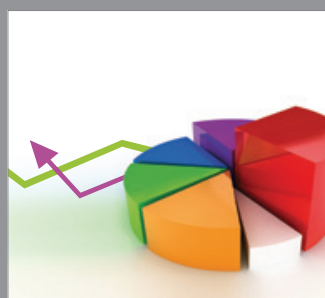

ournal of

Probability and Statistics

Promensencen
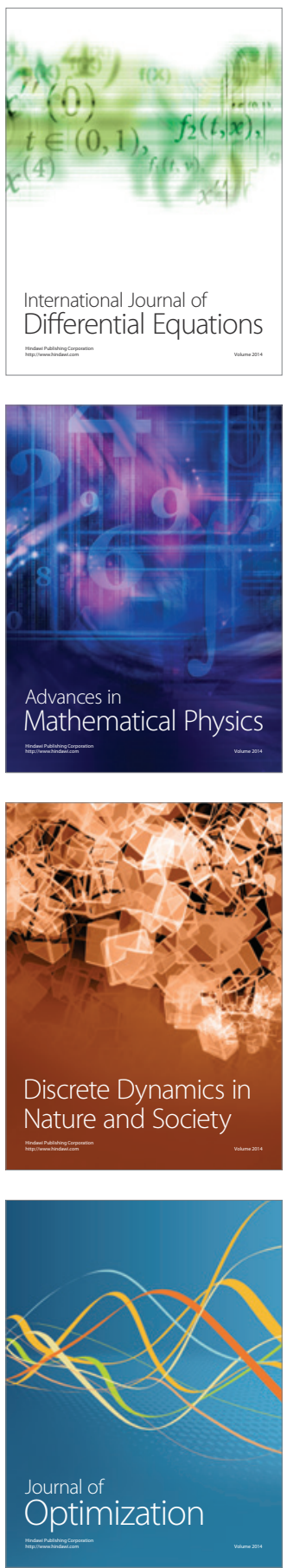